\title{
Optical probing of spin fluctuations of a single paramagnetic Mn atom in a semiconductor quantum dot
}

\author{
L. Besombes, ${ }^{1, *}$ Y. Leger, ${ }^{1}$ J. Bernos, ${ }^{1}$ H. Boukari, ${ }^{1}$ H. Mariette, ${ }^{1}$ J. P. Poizat, ${ }^{1}$ T. Clement, ${ }^{1}$ J. Fernández-Rossier, ${ }^{2}$ and \\ R. Aguado ${ }^{3}$ \\ ${ }^{1}$ CEA-CNRS group "Nanophysique et Semiconducteurs," Institut Néel, CNRS, 23 Avenue des Martyrs, 38042 Grenoble, France \\ ${ }^{2}$ Departamento de Física Aplicada, Universidad de Alicante, San Vicente del Raspeig, Alicante 03690, Spain \\ ${ }^{3}$ Instituto de Ciencia de Materiales de Madrid, CSIC, Madrid 28049, Spain \\ (Received 19 December 2007; revised manuscript received 17 July 2008; published 26 September 2008)
}

\begin{abstract}
We analyzed the photoluminescence intermittency generated by a single paramagnetic spin localized in an individual semiconductor quantum dot. The statistics of the photons emitted by the quantum dot reflect the quantum fluctuations of the localized spin interacting with the injected carriers. Photon correlation measurements, which are reported here, reveal unique signatures of these fluctuations. A phenomenological model is proposed to quantitatively describe these observations, allowing a measurement of the spin dynamics of an individual magnetic atom at zero magnetic field. These results demonstrate the existence of an efficient spin-relaxation channel arising from a spin exchange with individual carriers surrounding the quantum dot. A theoretical description of a spin-flip mechanism involving spin exchange with surrounding carriers gives relaxation times in good agreement with the measured dynamics.
\end{abstract}

DOI: 10.1103/PhysRevB.78.125324

PACS number(s): 78.67.Hc, 75.75.+a, 78.55.Et

\section{INTRODUCTION}

The decrease in the structure size in semiconductor electronic devices and magnetic information storage devices has dramatically reduced the number of atoms necessary to process and store bits of information. Information storage on a single magnetic atom would be an ultimate limit. The performance of such memory elements will be governed by the quantum fluctuations of the localized spins. ${ }^{1}$ Diluted magnetic semiconductor (DMS) systems combining high-quality semiconductor structures and the magnetic properties of $\mathrm{Mn}$ impurities are good candidates for these ultimate spintronic devices. ${ }^{2}$ It has been shown that in a DMS with low magnetic atom concentration, the spin dynamics under magnetic field is ultimately controlled by the spin-lattice coupling. ${ }^{3,4}$ An extrapolation of the spin dynamics measurements in bulk DMS suggests that a long spin-relaxation time in the millisecond range could be expected for an isolated Mn spin. ${ }^{5}$ However, despite the recent development of different successful techniques to address a single spin, ${ }^{6,7}$ such dynamics has never been directly observed.

Growth and optical addressing of DMS quantum dots (QDs) containing a single magnetic atom were achieved recently. ${ }^{8,9}$ When a Mn-dopant atom is included in a II-VI $\mathrm{QD}$, the spin of the optically created electron-hole pair interacts with the five $d$ electrons of the Mn (total spin $M=5 / 2$ ). This leads to a splitting of the once simple photoluminescence (PL) spectrum of an individual QD into six $(2 M+1)$ components. This splitting results from the spin structure of the confined holes, which are quantized along the QDs' growth axis with their spin component taking only the values $J_{z}= \pm 3 / 2$. The hole-Mn exchange interaction reduces to an Ising term $J_{z} M_{z}$ and shifts the emission energy of the QD depending on the relative projection of the Mn and hole spins. As the spin state of the Mn atom fluctuates during the optical measurements, the six lines are observed simultaneously in time-averaged PL spectrum. The intensities of the lines reflect the probability for the Mn to be in one of its six spin components and the emitted photon is a probe of the spin state of the Mn when the exciton recombines.

In this paper, we show how we can use the statistics of the photons emitted by a single Mn-doped QD to probe the spin dynamics of a magnetic atom interacting with both confined and free carriers. We performed correlations of the PL intensity emitted by individual lines of an isolated Mn-doped QD. In these start-stop experiments, ${ }^{10}$ the detection of the first photon indicates by its energy and polarization that the $\mathrm{Mn}$ spin has a given orientation. The detection probability of a second photon with the same energy and polarization is then proportional to the probability of conserving this spin state. The time evolution of this intensity correlation signal is a probe of the spin dynamics of the Mn atom. The autocorrelation signal displays a bunching effect revealing a PL intermittency. This intermittency results from fluctuations of the $\mathrm{Mn}$ spin. Correlation of the intensity emitted by opposite spin states of the exciton-Mn complex (namely, cross correlation) presents an antibunching at short delays. The characteristic time of this antibunching corresponds to the spintransfer time between the two degenerated spin states. A thermalization on the exciton-Mn complex is directly evidenced by the energy and temperature dependences of the correlation curves. The measured single Mn spin-relaxation times are in the range of $20 \mathrm{~ns}$ and are strongly influenced by the injection of carriers in the vicinity of the QDs. Mn spin flips induced by the injection of carriers in and around the QD are theoretically described. These scattering processes give relaxation times in good agreement with the measured dynamics.

\section{SAMPLES AND EXPERIMENTS}

Single Mn atoms are introduced in CdTe/ZnTe QDs during their growth by molecular-beam epitaxy adjusting the density of Mn atoms to be roughly equal to the density of 

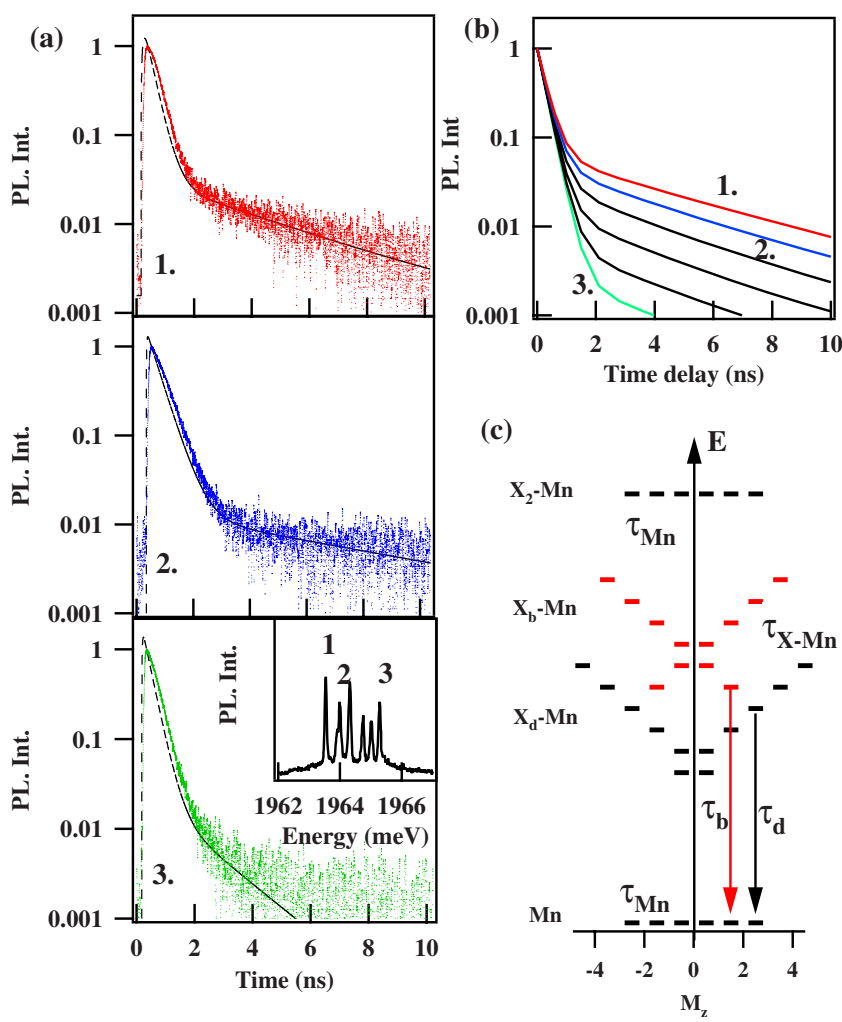

FIG. 1. (Color online) (a) Experimental time-resolved PL spectra recorded on three different energy lines (labeled 1,2, and 3) of an X-Mn complex. The inset shows the corresponding timeintegrated PL spectrum. (b) PL decay time calculated using the parameters $T=5 \mathrm{~K}, \tau_{b}=280 \mathrm{ps}, \tau_{d}=8 \mathrm{~ns}$, and $\tau_{\mathrm{X}-\mathrm{Mn}}=25 \mathrm{~ns}$. (c) Energy levels involved in the rate-equation model described in the text and displayed as functions of their total angular momentum $S_{z}$.

QDs. ${ }^{9}$ The statistics of the photons emitted by individual Mn-doped QDs were analyzed using a combination of a lowtemperature $(5 \mathrm{~K})$ scanning optical microscope and a Hanbury Brown and Twiss (HBT) setup for photon-correlation measurements. ${ }^{10}$ High refractive index hemispherical solid immersion lens were used to enhance the collection of the single-dot emission. The PL was quasiresonantly excited with a tunable continuous-wave $(\mathrm{CW})$ dye laser or by picosecond pulses from a doubled optical parametric oscillator and collected through aluminum shadow masks. ${ }^{11}$ The circularly polarized collected light was spectrally dispersed by a 1 $\mathrm{m}$ monochromator before being detected in the HBT setup or by a single fast avalanche photodiode (time resolution of 40 ps) for time-resolved measurements. The time resolution of the HBT setup was about 500 ps. Under our experimental conditions with count rates of a few kilohertz the measured photon pair distribution yields after normalization of the intensity autocorrelation function $g^{(2)}(\tau)$.

\section{EXCITON-MN SPIN FLIPS}

A signature of the Mn spin dynamics can be observed first in the PL decay of the X-Mn complex. Figure 1 presents the PL decay of three different transitions of the X-Mn complex. These transitions present a biexponential decay. The fast component corresponds to the radiative lifetime of the exciton as already measured in nonmagnetic QDs. The long component is associated with the existence of the dark excitons. ${ }^{12,13}$ Direct recombination of the dark exciton can be observed in some Mn-doped QDs because of a slight admixture of the bright states with the dark ones induced by a valence-band mixing. However, the dark excitons mainly contribute to the signal by undergoing a spin flip to become bright excitons, which decay radiatively. The PL decay is then determined by both radiative decay and exciton spin flips. The exciton decay, and particularly the amplitude of the slow component, depends strongly on the energy level observed. For the high-energy lines, the slow component makes a negligible contribution to the time-integrated signal. Conversely, for the low-energy lines, the secondary component makes a significant contribution while the primary lifetime remains constant. In this regime, the secondary lifetime can be associated either with the dark exciton lifetime or with the exciton spin-flip time.

To extract these two parameters from the PL decay curves, we compare the experimental data with a rateequation model describing the time evolution of the population of the $24 \mathrm{X}-\mathrm{Mn}$ spin levels [Fig. 1(c)] after the injection of a single exciton. ${ }^{14}$ Different spin-flip times are expected depending on whether the transitions occur with or without conservation of the energy or of the total spin. However, we consider in first approximation that the spin flips among the $\mathrm{X}-\mathrm{Mn}$ states can be described by a single characteristic time $\tau_{\mathrm{X}-\mathrm{Mn}}$. We consider that at finite temperature, the intraband relaxation rates $\Gamma_{\gamma \rightarrow \gamma^{\prime}}$ between the different spin states of the exciton-Mn complex depend on the energy separation $E_{\gamma \gamma^{\prime}}$ $=E_{\gamma^{\prime}}-E_{\gamma}$. Here we use $\Gamma_{\gamma \rightarrow \gamma^{\prime}}=1 / \tau_{\mathrm{X}-\mathrm{Mn}}$ if $E_{\gamma \gamma^{\prime}}<0$ and $\Gamma_{\gamma \rightarrow \gamma^{\prime}}=1 / \tau_{\mathrm{X}-\mathrm{Mn}} e^{-E_{\gamma \gamma^{\prime}} / k_{B} T}$ if $E_{\gamma \gamma^{\prime}}>0 .{ }^{14}$ This describes a partial thermalization among the 24 levels of the X-Mn system during the lifetime of the exciton (bright or dark). In this model, we also neglect the influence of the valence-band mixing on the oscillator strength ${ }^{15}$ and consider that all the excitonic bright (dark) states have the same lifetime $\tau_{b}\left(\tau_{d}\right)$. Because of an efficient hole spin flip during the phononassisted relaxation of the unpolarized injected carriers, we consider that the excitons with spins \pm 1 and \pm 2 are excited with the same probability at $t=0$. Only optical transitions conserving the $\mathrm{Mn}$ spin are considered. The calculation shows that changing $\tau_{d}$ mainly influences the characteristic time of the long decay component whereas changing $\tau_{\mathrm{X}-\mathrm{Mn}}$ affects also the amplitude of this component. The PL decay curves can be reproduced well by this rate-equation model using $\tau_{b}=280 \mathrm{ps}, \tau_{d}=8 \mathrm{~ns}$, and $\tau_{\mathrm{X}-\mathrm{Mn}}=25 \mathrm{~ns}$ [Fig. 1(b)]. The value of $\tau_{d}$ controls the decay time of the long component whereas $\tau_{\mathrm{X}-\mathrm{Mn}}$, which is larger than $\tau_{d}$, reproduces the emission energy dependence of the amplitude of the long component very well.

Within the X-Mn complex, the relaxation time between different spin states $\tau_{\mathrm{X}-\mathrm{Mn}}$ can be affected by the spin-orbit, carrier-phonon, and exchange interactions ${ }^{14}$ that affect the exciton. In QDs, the electron-spin relaxation is longer than the radiative lifetime and is ultimately controlled by random fluctuations of the nuclear spins. ${ }^{16}$ The hole spin relaxation is mainly controlled by the interaction with phonons ${ }^{17}$ and can be faster explaining the observed partial thermalization of 

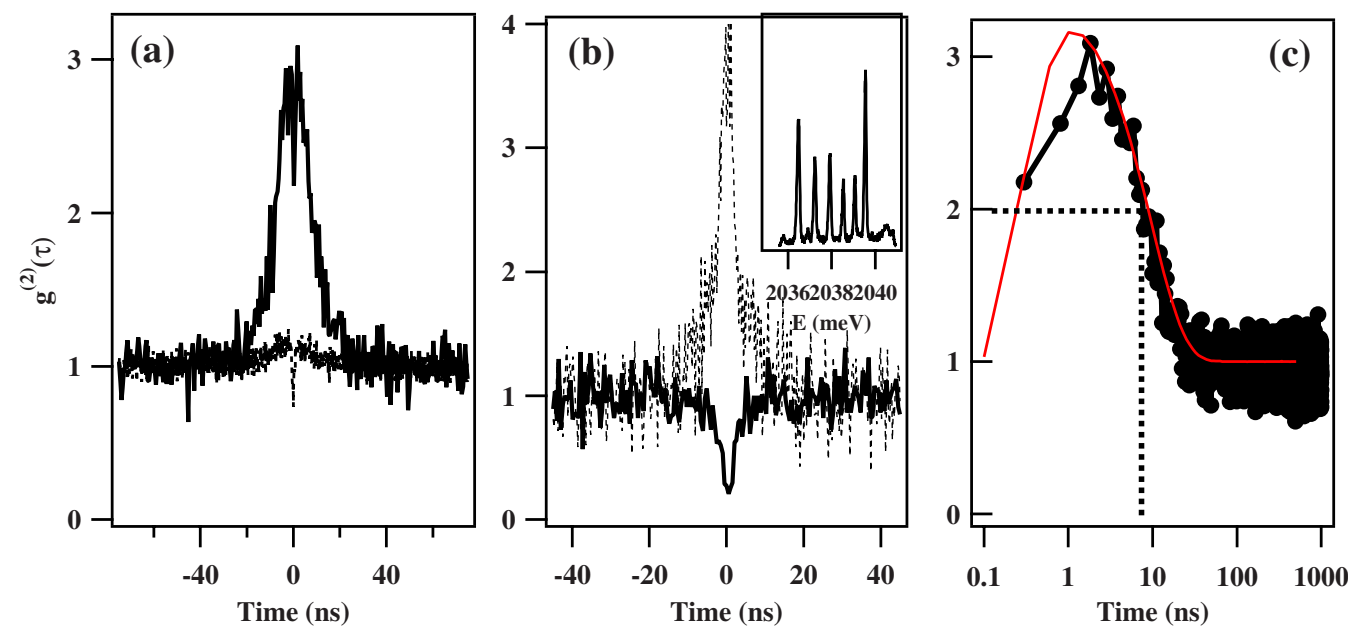

FIG. 2. (Color online) (a) Autocorrelation function of the intensity collected in $\sigma+$ polarization on the low-energy line of the X-Mn complex (solid line) and on the overall PL spectra (dotted line). (b) Circularly polarized cross-correlation (solid line) and autocorrelation functions (dotted line) on the same line as in (a) but for a larger excitation intensity. The inset shows the PL spectrum of the corresponding QD. (c) Experimental autocorrelation and theoretical functions calculated with the rate-equation model described in the text with parameters $T=5 \mathrm{~K}, \tau_{b}=280 \mathrm{ps}, \tau_{d}=8 \mathrm{~ns}, \tau_{\mathrm{X}-\mathrm{Mn}}=25 \mathrm{~ns}, \tau_{\mathrm{Mn}}=50 \mathrm{~ns}$, and $g=0.05 \times 10^{-3} \mathrm{ps}^{-1}$.

the X-Mn complex during the lifetime of the ground-state exciton.

\section{FLUCTUATIONS OF AN ISOLATED MN SPIN}

To directly observe the time fluctuations of the Mn spin interacting with the injected carriers, we analyzed the statistics of the photons emitted by a Mn-doped QD. This statistics can be deduced from an intensity correlation function of the QD emission. Figure 2(a) shows the intensity correlation function $g^{(2)}(\tau)$ of the circularly polarized $(\sigma+)$ low-energy line of a Mn-doped QD compared with the correlation function obtained for the overall PL of the QD. The autocorrelation function obtained for all the photons emitted by the QD is characteristic of a single-photon emitter with a dip at short delay. The width of this antibunching signal is given by the lifetime of the emitter and the generation rate of excitons and its depth is limited by the time resolution of the HBT setup. A similar experiment performed on one of the single line of the X-Mn complex still presents a reduced coincidence rate near $\tau=0$, but it is mainly characterized by a large bunching signal with a half width at half maximum (HWHM) of about $10 \mathrm{~ns}$. This bunching reflects an intermittency in the QD emission. This intermittency likely comes from the fluctuations of the Mn spin orientation.

To confirm this result, cross-correlation measurements were performed between different spin states of the X-Mn complex. Cross correlation of the $\sigma+$ and $\sigma$ - photons emitted by the low-energy line [Fig. 2(b)] shows an antibunching with $g^{(2)}(0)=0.2$ and a HWHM of about 5 ns. These two different behaviors, namely, the bunching of the autocorrelation signal and the antibunching of the cross-correlation signal, demonstrate unambiguously that the statistic of the QD emission is completely governed by the Mn spin fluctuations. Whereas the autocorrelation probes the time dependence of the probability for the spin of the $\mathrm{Mn}$ to be conserved $\left[M_{z}\right.$ $=+5 / 2$ at $\tau=0$ in Fig. 2(a)], the cross correlation presented in Fig. 2(b) is a probe of the spin transfer between $+5 / 2$ and $-5 / 2$.

As observed in the time-resolved PL measurements, fluctuations of the Mn spin occur during the lifetime of an exciton. However, they can also take place when the QD is empty. As the spin-relaxation rate of the $\mathrm{Mn}$ is expected to be influenced by the presence of carriers in the QD, we have to consider two relaxation times: $\tau_{\mathrm{Mn}}$ for an empty dot and $\tau_{\mathrm{X}-\mathrm{Mn}}$ for a dot occupied by an exciton. Their relative contributions to the observed effective relaxation time will depend on the generation rate of excitons. The rate-equation model described previously can be extended to extract an order of magnitude of the parameters $\tau_{\mathrm{Mn}}$ and $\tau_{\mathrm{X}-\mathrm{Mn}}$ from the correlation experiments. Six biexciton states are added to the $24(\mathrm{X}-\mathrm{Mn})+6(\mathrm{Mn})$ level system [see Fig. 1(c)]. A continuous generation rate $g$ is considered to populate the exciton and biexciton states. The initial state of the system is fixed on one of the six Mn ground states and one monitors the time evolution of the population of the corresponding bright $\mathrm{X}-\mathrm{Mn}$ state. When normalized to one at a long time, this time evolution accounts for the correlation function of the transition associated with the considered X-Mn level.

The time evolution of the correlation function calculated using this model is presented in Fig. 2(c) and compared with the experimental data. At low generation rate, when the average time between two injected excitons is longer than any spin-relaxation rate, $\tau_{\mathrm{Mn}}$ and $\tau_{\mathrm{X}-\mathrm{Mn}}$ have distinguishable effects on the calculated correlation curves. In average, the relaxation of the $\mathrm{Mn}$ alone (controlled by $\tau_{\mathrm{Mn}}$ ) is only perturbed by the injection of the exciton used to probe the Mn spin projection. During the lifetime of this exciton, the system relaxes with the relaxation rate $\tau_{\mathrm{X}-\mathrm{Mn}}$. This produces a relaxation of the $\mathrm{Mn}$ spin proportional to the ratio of $\tau_{\mathrm{X}-\mathrm{Mn}}$ and the exciton lifetime. A reduction in $\tau_{\mathrm{X}-\mathrm{Mn}}$ then reduces the amplitude of the bunching curve expected for a Mn alone (because of the six available spin states, the maximum amplitude of the bunching should be six) without significantly 

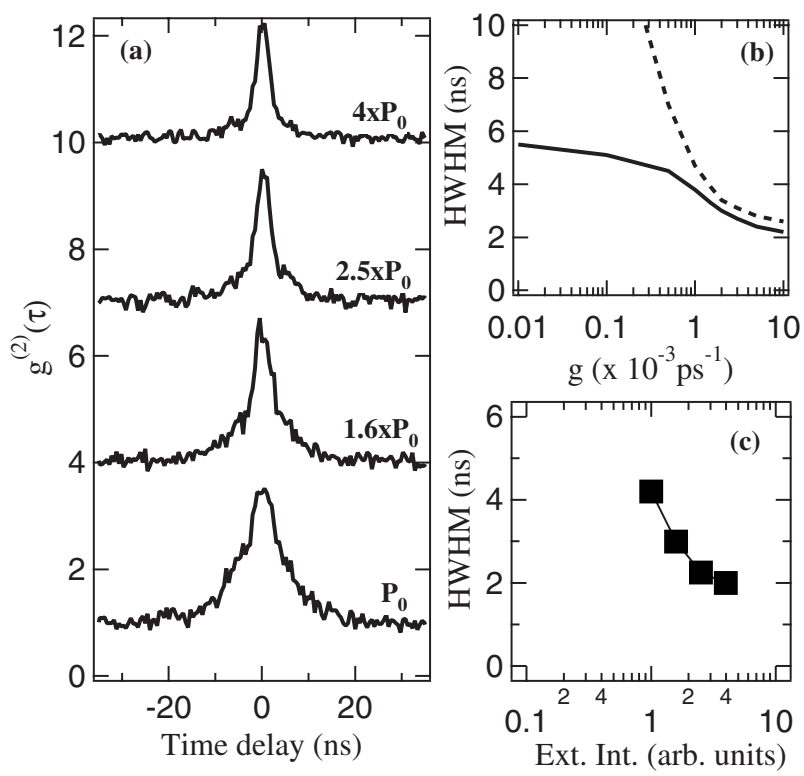

FIG. 3. (a) Power dependence of the autocorrelation function of the high-energy line of an X-Mn complex. (b) Calculated power dependence of the HWHM of the autocorrelation function of the high-energy line. The parameters used in the model are: $\tau_{b}=280 \mathrm{ps}, \tau_{d}=8 \mathrm{~ns}, \tau_{\mathrm{X}-\mathrm{Mn}}=20 \mathrm{~ns}$, and $\tau_{\mathrm{Mn}}=40 \mathrm{~ns}$ (plain line) or $\tau_{\mathrm{Mn}}=4 \mu$ s (dotted line). (c) Experimental HWHM of the autocorrelation signals presented in (a).

changing its width that is controlled by $\tau_{\mathrm{Mn}}$. With the generation rate used in the measurements of Fig. 2(a) (a generation rate of about $g=0.05 \times 10^{-3} \mathrm{ps}^{-1}$ can be deduced from the ratio of the exciton and biexciton amplitudes), $\tau_{\mathrm{X}-\mathrm{Mn}}$ mainly affects the height of the bunching signal whereas $\tau_{\mathrm{Mn}}$ preferentially controls its width. Then, with given values of $g, \tau_{b}$, and $\tau_{d}$, it is possible to extract a parameter pair $\left(\tau_{\mathrm{Mn}}, \tau_{\mathrm{X}-\mathrm{Mn}}\right)$ that reproduces the bunching and antibunching curves. The bright and dark exciton lifetimes were estimated from the PL decay curves, and the exciton generation rate can be estimated from the relative amplitudes of the exciton and biexciton emissions. ${ }^{18}$ For the data presented in Fig. 2(c), the best fit is obtained with $\tau_{\mathrm{X}-\mathrm{Mn}}=25 \mathrm{~ns}$ and $\tau_{\mathrm{Mn}}$ $=50 \mathrm{~ns}$. The X-Mn relaxation time obtained is consistent with the value deduced from the PL decay curves. The relaxation time of the Mn alone (empty dot) appears to be three orders of magnitude shorter than expected from the extrapolation of measurements in bulk-dilute CdMnTe under magnetic field. ${ }^{5}$

\section{CARRIER-INDUCED MN SPIN FLUCTUATIONS}

The observed Mn spin dynamics is not simply an intrinsic property of the localized $\mathrm{Mn}$ atom but depends on the optical excitation conditions. The power dependence of the correlation signal of the high-energy transition of an X-Mn complex is presented in Fig. 3(a). Increasing the excitation power significantly reduces the width of the correlation signal. This reduction has two origins. When carriers are injected in the QD under quasiresonant conditions (excitation on an excited state of the QD), increasing the carrier generation rate in-
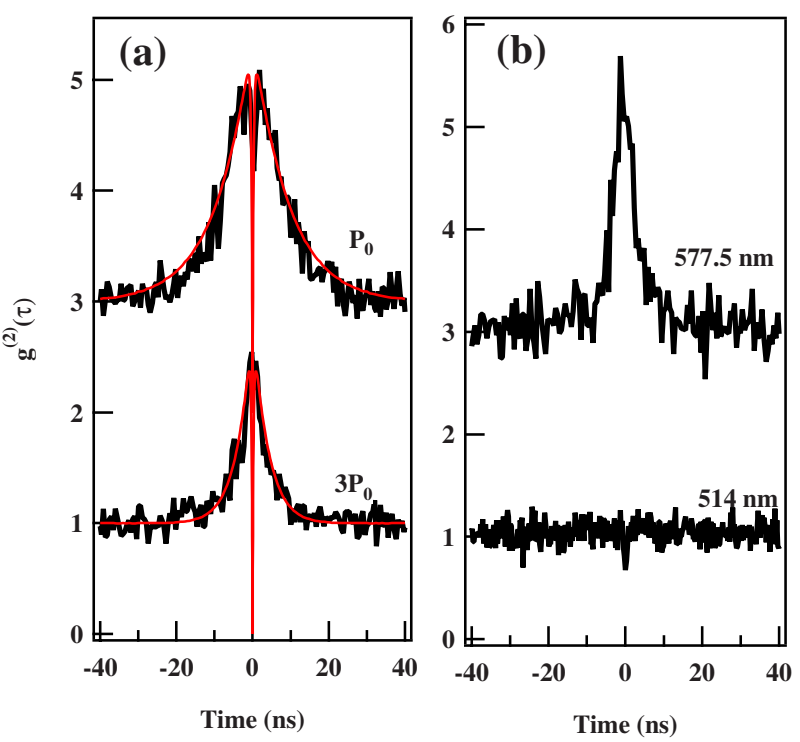

FIG. 4. (Color online) (a) Autocorrelation function on the lowenergy line of an X-Mn complex in $\sigma+$ polarization for excitation intensities $P_{0}$ and $3 P_{0}$. Theoretical curves are presented in red. A reduction in $\tau_{\mathrm{X}-\mathrm{Mn}}(=15 \mathrm{~ns})$ and $\tau_{\mathrm{Mn}}(=20 \mathrm{~ns})$ has to be included to describe the high-excitation intensity autocorrelation curve. (b) Autocorrelation function on the low-energy line of an X-Mn complex in $\sigma+$ polarization for two different excitation conditions: resonant on an excited state $(577.5 \mathrm{~nm})$ and nonresonant $(514 \mathrm{~nm})$.

creases the probability of finding the QD occupied by an exciton. With the spin-relaxation time being shorter for an occupied dot than for an empty dot, the Mn spin fluctuates faster and the width of the autocorrelation curve decreases. This effect is illustrated by the power dependence of the HWHM of the calculated and experimental correlation curves presented in Figs. 3(b) and 3(c), respectively. At high generation rate, the width of the correlation signal is controlled by $\tau_{\mathrm{X}-\mathrm{Mn}}$ whereas at low excitation the photon statistics are ultimately determined by the spin fluctuations of the $\mathrm{Mn}$ alone. The width of the calculated correlation curves saturates at low excitation. This maximum width is controlled by $\tau_{\mathrm{Mn}}$. In the experiments, this saturation is not observed due to the limit in the accessible excitation-power range.

However, this process is not sufficient to explain the observed excitation-power dependence of the correlation signal in all the investigated QDs. For instance, to reproduce the power dependence presented in Fig. 4(a), one also has to reduce the spin-relaxation times $\tau_{\mathrm{Mn}}$ and $\tau_{\mathrm{X}-\mathrm{Mn}}$ at highexcitation intensity. In Fig. 4(a) the best fit at high-excitation power is obtained with $\tau_{\mathrm{Mn}}=25 \mathrm{~ns}$ and $\tau_{\mathrm{X}-\mathrm{Mn}}=15 \mathrm{~ns}$ whereas at low excitation $\tau_{\mathrm{Mn}}=50 \mathrm{~ns}$ and $\tau_{\mathrm{X}-\mathrm{Mn}}=25 \mathrm{~ns}$. This reduction in the relaxation time likely comes from the spinspin coupling with carriers injected in the surroundings of the QD through the background absorption observed in photoluminescence excitation spectra of this individual QD. ${ }^{19}$

The influence of the free carriers on the spin-relaxation rate is shown by the correlation signals obtained on the same X-Mn transition for two different excitation wavelengths [Fig. 4(b)]: resonant on an excited state $(577.5 \mathrm{~nm})$ and nonresonant in the ZnTe barriers $(514 \mathrm{~nm})$. These two signals are 
recorded with the same photon-count rate suggesting a similar occupation rate of the QD. The characteristic bunching signal observed under quasiresonant excitation completely disappears when the excitation energy is tuned above the wetting-layer absorption. As already observed in DMS quantum wells, this behavior reflects the extreme sensitivity of the localized Mn spin to the spin-spin coupling with the free carriers or the carriers relaxing in the QD. ${ }^{20}$

For an isolated Mn atom, the spin relaxation $\tau_{\mathrm{Mn}}$ comes only from the spin-lattice interaction, ${ }^{21}$ and a long spinrelaxation time is expected. As we will discuss in Sec. VI, the Mn spin dynamics can be modified significantly by the presence of free carriers, which are strongly coupled with both the magnetic atom and the phonons. These free carriers serve as a bypass channel for the slow direct spin-lattice relaxation.

\section{THERMALIZATION OF THE EXCITON-MN COMPLEX}

The X-Mn complex is also significantly coupled to the phonon bath. A partial thermalization of the X-Mn system appears directly in the amplitude of the correlation curves obtained on different energy levels of the X-Mn system [Fig. 5(a)] as well as in cross-correlation measurements [Figs. 5(b) and $5(\mathrm{c})]$. A finite temperature enhances the probability of the spin flips involving an energy loss. This introduces a dissymmetry in the spin-relaxation channels of the X-Mn complex. The consequence of this dissymmetry in the relaxation process is an energy dependence of the amplitude of the correlation signal. This is illustrated by the correlation curves obtained in the high- $(|-1,-5 / 2\rangle)$ and low-energy states $(|+1,-5 / 2\rangle)$ of an X-Mn complex [Fig. 5(a)]. For the high-energy state, all the relaxation transitions within the $\mathrm{X}-\mathrm{Mn}$ complex take place with an energy loss: The leakage probability is then at a maximum and the probability for this state to be repopulated by spin flips from low-energy states is very weak. A large bunching signal is then observed [Fig. 5(a)]. On the opposite, the low-energy level can be populated by a transfer from the high-energy states, and some relaxation channels involving an absorption of energy are blocked at low temperature. The associated bunching signal is weaker [Fig. 5(a)].

This thermalization process directly appears if a cross correlation of the intensity emitted by a low- and a high-energy levels is performed. Figure 5(c) shows the correlation of the photons emitted by $|-1,-5 / 2\rangle$ (high-energy line) and $\mid+1$, $-5 / 2\rangle$ (low-energy line). At low-excitation intensity, this correlation signal presents a clear dissymmetry. This crosscorrelation measurement probes the time dependence of the probability of finding an exciton (either $|+1\rangle$ or $|-1\rangle$ ) coupled with the Mn spin in the state $M_{z}=-5 / 2$. At positive time delay, $g^{2}(\tau)$ gives the probability to find the system in the state $|+1,-5 / 2\rangle$ knowing that at $\tau=0$ the Mn spin projection is $M_{z}=-5 / 2$ (detection of a photon from $|-1,-5 / 2\rangle$ ). The situation is reversed for the negative delay where a photon from $|+1,-5 / 2\rangle$ acts as the trigger in the start-stop measurement, and $g^{2}(\tau)$ gives the probability for the system to be in the high-energy state $|-1,-5 / 2\rangle$. The dissymmetry in the
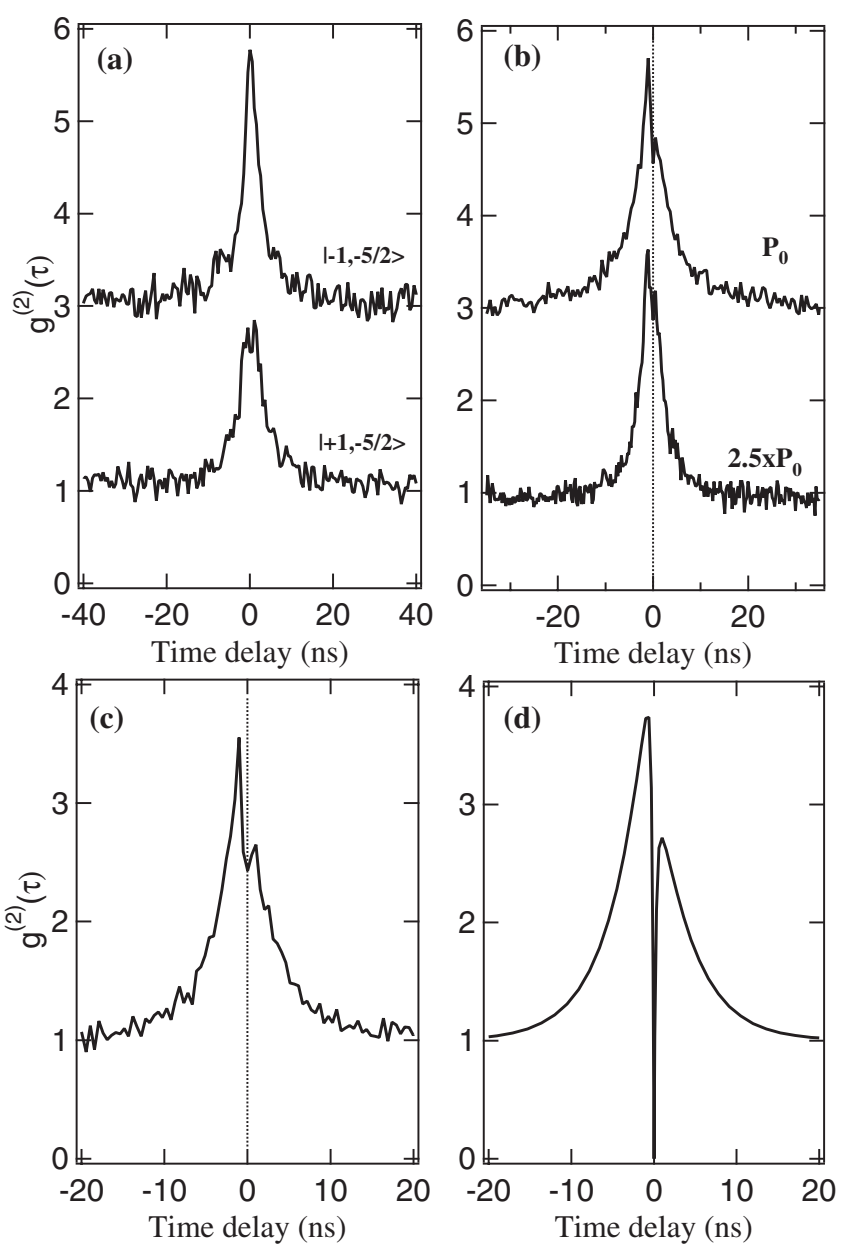

FIG. 5. (a) Autocorrelation function of the emission intensity of the high- (upper trace) and low- (lower trace) energy lines of an $\mathrm{X}-\mathrm{Mn}$ complex recorded in the same circular polarization. (b) Cross-correlation function of the emission intensity of the high- and low-energy lines recorded in $\sigma+$ and $\sigma$ - polarizations, respectively, for two different excitation intensities. Detail of the (c) experimental and (d) calculated cross-correlation functions.

cross-correlation curve reflects the difference in the spinrelaxation channels available for the high- $(|-1,-5 / 2\rangle)$ and the low- $(|+1,-5 / 2\rangle)$ energy X-Mn states.

The dissymmetry in the relaxation processes is influenced by the excitation intensity: as observed in the PL decay measurements presented in Fig. 1, the low-energy bright exciton states can be efficiently populated by spin flips from the dark exciton states reducing the effective population loss of these states and consequently reducing the amplitude of the photon bunching. Increasing the excitation intensity decreases the effective lifetime of the dark excitons because of the formation of the biexciton. ${ }^{22}$ This opens an efficient spin-relaxation channel for the low-energy bright X-Mn states: Once a dark exciton has been created after a spin flip, it is quickly destroyed by the injection of a second exciton with the formation of a biexciton. It can no longer flip back to the lowenergy bright state. This effect stops the refilling process and consequently increases the amplitude of the bunching signal. As observed in Fig. 5(b), increasing the excitation intensity decreases the difference in the amplitude of the correspond- 


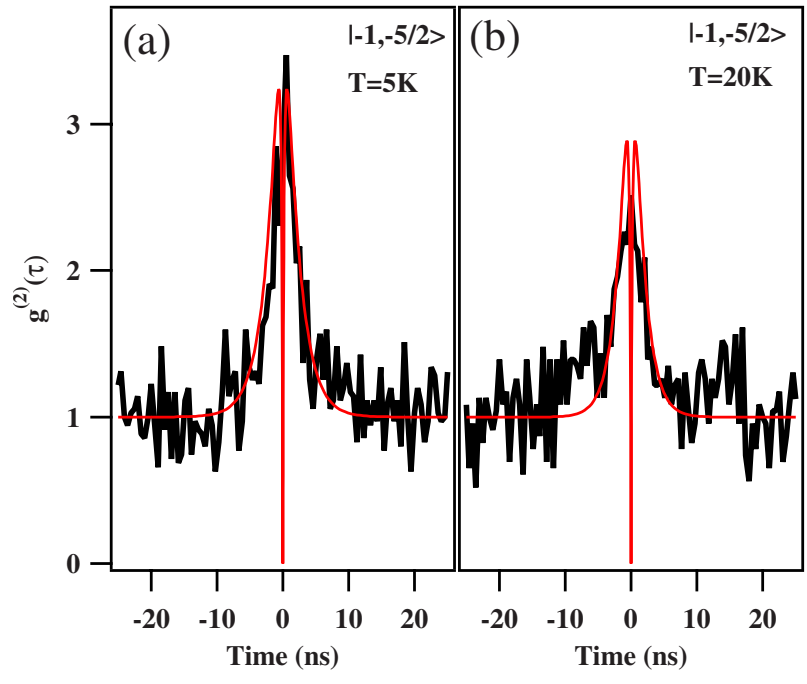

FIG. 6. (Color online) Intensity autocorrelation function of the high-energy line $|-1,-5 / 2\rangle$ of an X-Mn complex recorded in the same excitation conditions at (a) $T=5$ and (b) $20 \mathrm{~K}$. The theoretical curves (solid line) are obtained with the same set of parameters: $\tau_{\mathrm{X}-\mathrm{Mn}}=10 \mathrm{~ns}, \quad \tau_{\mathrm{Mn}}=15 \mathrm{~ns}, \tau_{b}=280 \mathrm{ps}, \quad \tau_{d}=8 \mathrm{~ns}$, and $g=0.1$ $\times 10^{-3} \mathrm{ps}^{-1}$.

ing bunching signal of the low- and high-energy lines.

As shown in Fig. 6, these spin-relaxation processes are also influenced by the lattice temperature. The effect is especially pronounced for the high-energy states. Increasing the temperature allows a refilling of these levels by a transfer of population from the low-energy states, decreasing the amplitude of the bunching signal.

\section{MODEL FOR THE MN SPIN RELAXATION}

In this section we discuss two Mn spin-relaxation mechanisms in the optical ground state. The short $\left(\tau_{\mathrm{Mn}} \simeq 20 \mathrm{~ns}\right)$ spin-relaxation time inferred from our photon-correlation measurements cannot be accounted for by Mn-Mn spin interaction, which is considered the most efficient spinrelaxation mechanism in II-VI semiconductor. Since this is a short-range interaction, ${ }^{23,24}$ the $\mathrm{Mn}$ spin-relaxation time $T_{1 M}$ increases exponentially as the density of $\mathrm{Mn}$ goes down. ${ }^{5}$ Thus, the spin relaxation of a single Mn atom inside a quantum dot with $20 \times 10^{3}$ atoms, if generated by Mn-Mn coupling, should be in the range of microseconds. Thus, some other spin-relaxation mechanism is at play. Here we study the Mn spin relaxation due to exchange coupling to photocarriers that occupy extended states in the wetting layer (WL). The Mn is assumed to lie in the wetting layer at a location where both quantum dot and extended states are finite. In particular, the QD states are expected to peak in the plane that separates the wetting layer and the QD. Mn atoms located in that region are more strongly coupled to the QD exciton.

We consider two independent mechanisms: (i) exchange coupling with carriers in extended WL states and (ii) exchange coupling with carriers that scatter from the extended WL states to confined QD states, which exchange with the
$\mathrm{Mn}$ and then return to the WL. The first mechanism has been considered before in the context of Mn spin relaxation interacting with quantum-well carriers ${ }^{25,26}$ and is identical to Korringa mechanism for nuclear-spin relaxation due to spin flip with itinerant electrons in metals. The second mechanism involves single charge tunneling in and out of the dot. The QD confined states remain empty except for some intervals during which a single carrier tunnels in and out from the optically excited wetting layer. Once in the QD state, the carrier can give (or take away) one unit of spin to Mn. Both the Korringa and the charge-fluctuation mechanisms require several tunneling and exchange events, involving different carriers, to relax the Mn spin from an initial situation where it is known to take the value $M_{z}=+5 / 2$ to a final situation of thermal equilibrium where the six Mn spin orientations are equally likely.

The proposed charge-fluctuation induced spin-relaxation mechanism is in line with our previous works s, $^{2,27-29}$ whose central claim is the very strong dependence of the spin properties of Mn-doped quantum dots on the addition of a single carrier. It is also motivated by the observation of a weak contribution of the positive trion PL signal, which implies that, under our experimental conditions, holes are captured by the QD.

\section{A. Formalism}

We use a Bloch-Redfield master-equation approach, ${ }^{30}$ which tracks the dynamical evolution of the density matrix of the quantum dot ground states, corresponding to the six $\mathrm{Mn}$ spin orientations under the influence of the reservoir of carriers. The diagonal terms of the density matrix represent the probability of finding the $\mathrm{Mn}$ spin in a given spin projection. Their dynamics is given by the master equation

$$
\frac{d p_{M}}{d t}=-\left(\sum_{M^{\prime}} \Gamma_{M \rightarrow M^{\prime}}\right) p_{M}+\sum_{M} \Gamma_{M^{\prime} \rightarrow M} p_{M^{\prime}},
$$

with the initial condition that at $t=0$, the probability of finding the Mn spin with $M_{z}=+5 / 2$ is $p_{+5 / 2}=1$. The evolution of $p_{+5 / 2}(t)$ and $p_{-5 / 2}(t)$ is directly associated with the autocorrelation and cross-correlation measurements. The rates in the master equation depend on the Hamiltonian of the system, which is the sum of the QD Hamiltonian, the WL carrier Hamiltonian, and the QD-WL coupling: $\mathcal{H}=\mathcal{H}_{0}+\mathcal{H}_{\text {res }}+\mathcal{V}$.

$\Gamma_{M \rightarrow M^{\prime}}$ is the scattering rate between the eigenstate $M$ and state $M^{\prime}$ of $\mathcal{H}_{0}$ induced by the coupling $\mathcal{V}$. The rates are given by the statistical average over initial reservoir states and sum over final reservoir states of the Fermi-golden-rule rate associated with $\mathcal{V} .^{30}$ They depend on the mechanism under consideration, either direct exchange or charge fluctuation. Since our experimental data indicate that hole confinement is much weaker than electron confinement, we consider that the carriers that couple to the $\mathrm{Mn}$ when there is no exciton in the dot are holes. Our theory results can easily be adapted for the case of electrons. The number of states $M$ in the master equation also depends on the mechanism. In the case of charge-fluctuation mechanism we need to keep track of the 6 neutral states and the 12 states of the dot with one hole inside. In the case of the Korringa relaxation, only the 
six states corresponding to the "empty-dot" Mn spin are included in the master equation.

The QD Hamiltonian is

$$
\mathcal{H}_{0}=J\left[\tau^{z} \hat{\mathbf{M}}^{z}+\frac{\epsilon_{h}}{2}\left(\tau^{+} \hat{\mathbf{M}}^{-}+\tau^{-} \hat{\mathbf{M}}^{+}\right)\right]+D\left(\hat{\mathbf{M}}^{z}\right)^{2},
$$

where $J=\beta\left|\psi_{\mathrm{QD}}\left(\vec{r}_{I}\right)\right|^{2} n_{d}$ is the Mn QD hole coupling, $\beta \simeq 60 \mathrm{eV}^{3}$ is the Mn-hole exchange coupling constant of CdTe, $\left|\psi_{\mathrm{OD}}(\vec{r})\right|^{2}$ is the QD hole envelope function, and $n_{d}=0,1$ counts the number of holes in the dot. The Mn spin operators are $\hat{\mathbf{M}}^{z, \pm}$ and $\tau^{z, \pm}$ are the Pauli matrices acting on the hole space. We consider antiferromagnetic hole-Mn coupling. $\epsilon_{h}$ is the dimensionless parameter that accounts for the reduction in the spin-flip interaction due to spin-orbit coupling. ${ }^{2,28}$

Notice that the exchange coupling of the Mn to the quantum dot (QD) fermion is only relevant in the chargefluctuation mechanism for which $n_{d}$ changes between zero and one. For the Korringa mechanism $n_{d}=0$ and Mn is only exchange coupled to carriers in extended WL states. The $D\left(\hat{\mathbf{M}}^{z}\right)^{2}$ term in $\mathcal{H}_{\mathrm{QD} 0}$ describes the strain-induced anisotropy, which has been observed in strained $(\mathrm{Cd}, \mathrm{Mn}) \mathrm{Te}$ layers. ${ }^{31}$ This term is negligible when the Mn interacts with a QD hole but is not when we consider the Korringa relaxation for which no carrier occupies the QD state. The term $\mathcal{H}_{\text {res }}$ $=\sum_{\mathbf{k}, \nu} \epsilon_{\mathbf{k}, \nu} c_{\mathbf{k}, \nu}^{\dagger} c_{\mathbf{k}, \nu}$ describes the delocalized carriers in states with momentum $\mathbf{k}$ and band index (including the spin) $\nu$.

Each of the two relaxation mechanisms considered here has its own dot-reservoir coupling. In the Korringa mechanism we assume that there is some overlap between the WL extended states and the Mn spin. Ignoring the dependence on $\mathbf{k}$ of the spin matrix element $\epsilon\left(\mathbf{k}, \mathbf{k}^{\prime}\right)$, the coupling reads

$$
\mathcal{V} \simeq \frac{\beta \epsilon}{2 A}\left|\phi\left(z_{I}\right)\right|^{2} \sum_{\mathbf{k}, \mathbf{k}^{\prime}}\left(\hat{\mathbf{M}}^{(+)} c_{\mathbf{k}, \Downarrow}^{\dagger} c_{\mathbf{k}^{\prime}, \Uparrow}+\hat{\mathbf{M}}^{(-)} c_{\mathbf{k}, \Uparrow}^{\dagger} c_{\mathbf{k}, \Downarrow}\right)
$$

where $A$ is the area of the WL, $\left|\phi\left(z_{I}\right)\right|^{2}$ is the envelope part of the WL wave function evaluated at the Mn location, and $L$ is the WL width. $\epsilon_{h}$ could take different values for the QD and the WL.

In the charge-fluctuation mechanism the coupling between the reservoir and the dot is the sum of an operator that transfers one hole from the reservoir to the dot and its Hermitian conjugate, which takes the hole out from the dot and transfers it to the reservoir,

$$
\mathcal{V}=\sum_{\sigma}|\sigma\rangle\langle 0| \sum_{\mathbf{k}} V_{\mathbf{k}} c_{\mathbf{k}, \sigma}+\text { H.c. }
$$

The tunneling operator conserves the energy and the spin of the carrier. This kind of coupling has been considered recently to account for peculiar photoluminescence (PL) line shapes of self-assembled quantum dots in contact with electronic reservoirs. ${ }^{12,32}$ The mechanism would operate analogously if the transfer from the itinerant to the localized state is inelastic, but the timescale would be longer.
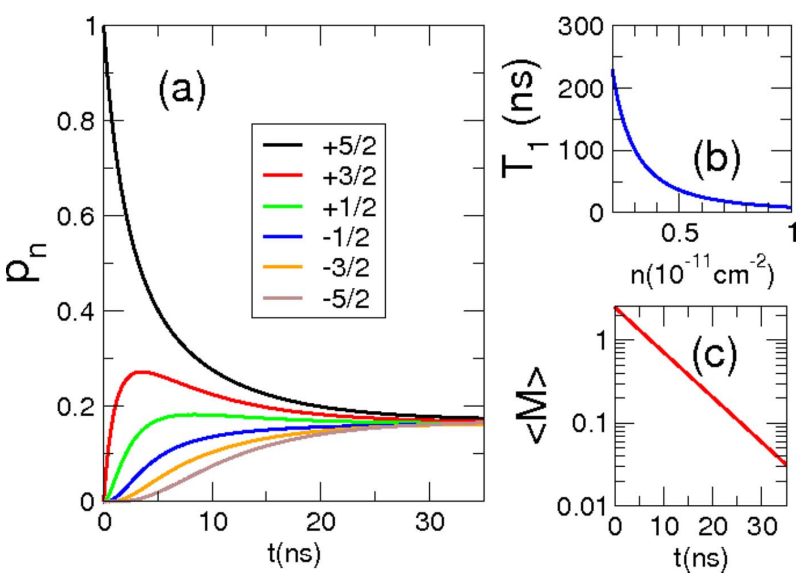

FIG. 7. (Color online) (a) $p_{n}$ for Mn spin states. These can be considered as the conditional probabilities that, having observed the Mn spin at $t=0$ in the state $M_{z}=+5 / 2$, a state with spin $M_{z}$ is observed at time $t$. (b) $T_{1 M}$ as a function of hole density (see text). (c) Average magnetization corresponding to panel (a) in logarithmic scale.

\section{B. Results}

\section{Mn spin relaxation due to exchange with WL carriers}

We discuss first the relaxation of the Mn spin due to exchange with WL carriers or Korringa mechanism. We assume a parabolic dispersion with effective mass $m^{*}$ for the holes, which yields a stepwise density of states. We assume holes are in a thermal state with an effective temperature $k_{B} T_{h}$ larger than the lattice and chemical potential $\mu$. The Korringa relaxation rate is proportional to the square of the density of states at the Fermi energy and the square of the exchange coupling constant. After some work we obtain the Mn spinflip rates between $M$ and $M^{\prime}$ eigenstates of $\mathcal{H}_{0}=D\left(\hat{\mathbf{M}}^{z}\right)^{2}$,

$$
\Gamma_{M \rightarrow M^{\prime}}^{ \pm}=\left(\frac{\beta \epsilon \eta m^{*}}{\pi L \hbar^{2}}\right)^{2} I(y, z)\left|\left\langle M\left|\hat{\mathbf{M}}^{( \pm)}\right| M^{\prime}\right\rangle\right|^{2},
$$

where we have approximated $\left|\phi\left(z_{I}\right)\right|^{2}=2 \eta / L, 0<\eta<1$ is a dimensionless parameter that accounts for the overlap of the WL states with the Mn, $y=\beta \mu$ is the chemical potential of the WL carriers, $z=\left(E_{M}-E_{M}^{\prime}\right) / k_{B} T_{h}$ is the change in energy of the Mn spin divided by the effective temperature of the WL carriers, and $I(y, z)=k_{B} T_{h} \frac{e^{y}}{e^{|z|}-1} \log \left[\frac{1+e^{y}}{1+e^{y-z \mid}}\right]$ for $z>0$ and $I(y, z)=e^{|z|} I(y,-z)$ for $z<0$.

The solution of the master equation, taking as initial condition $p_{+5 / 2}=1$, is shown in Fig. 7 . In the left panel we plot the evolution of the occupations of the six Mn spin projections along the growth axis. The decay of $p_{+5 / 2}$ is accompanied by a rise of the $+3 / 2$ state. This state is also connected with the $1 / 2$ state whose occupation starts to build. Interestingly, the population of the $+3 / 2$ states overshoots initially. The figure shows a "falling-domino" effect. They evolve toward thermal equilibrium. The average magnetization $\langle M\rangle(t)=\sum_{n=1,6} M_{n} p_{n}(t)$ decays according to 


$$
\langle M\rangle(t)=M_{0} e^{-t / T_{1 M}}
$$

in all the simulations performed. In the figure we take $T_{h}$ $\simeq 10 \mathrm{~K}, \epsilon=0.1, \eta=1, \mathcal{D}=7 \mu \mathrm{eV}, m^{*}=0.4, L=20 \AA$, and $n_{h}=1 \times 10^{11} \mathrm{~cm}^{-2}$ and we obtain $T_{1 M} \simeq 8 \mathrm{~ns}$. Thus, this mechanism is consistent with the Mn spin-relaxation time that we observe. In Fig. 7 we see how $T_{1 M}$ increases as the density of carriers decreases. The order of magnitude of $T_{1 M}$ is consistent with the calculations for $\mathrm{Mn}$ spin relaxation induced by scattering with electrons in quantum wells. ${ }^{25}$ There $T_{1 M} \simeq 2 \times 10^{3} \mathrm{~ns}$ is obtained in a quantum well with $L=80 \AA$. Taking into account that the electron-Mn coupling $\alpha$ is four times smaller than $\beta$ and the $L$ is four times bigger, the $T_{1 M}$ for holes should be at least $16 \times 10^{2}$ shorter.

Thus Korringa exchange with a sufficiently large density of photoholes might account for the Mn spin relaxation when the dot is in the optical ground state. We consider now a different mechanism, involving single-hole charging of the dot, motivated by the fact that the PL often shows a weak contribution of positive trions.

\section{Mn spin relaxation due to charge fluctuation in the dot}

The elementary process for spin relaxation due to charge fluctuation is a combination of three steps. First, a hole tunnels into the empty dot with the $\mathrm{Mn}$ in the spin state $M$. Second, the hole and the Mn exchange spin so that the Mn is now in the state $M \pm 1$. Third, the hole tunnels out of the dot. Thus, in the master equation we need to include both the six charge neutral states of the Mn-doped dot as well as the 12 states with one hole inside. The dissipative dynamics induced by $\mathcal{V}$ connects neutral states with charged states $(+)$ and vice versa $(-)$. The charging transition rates are given by

$$
\Gamma_{n, m}^{(+)}=\Gamma_{0} n_{F}\left(E_{n}-E_{m}\right)\left|\left\langle n\left|\sum_{\sigma}\right| 0\right\rangle\langle\sigma|| m\rangle\right|^{2}
$$

and the decharging rate

$$
\Gamma_{n, m}^{(-)}=\Gamma_{0}\left[1-n_{F}\left(E_{n}-E_{m}\right)\right]\left|\left\langle n\left|\sum_{\sigma}\right| 0\right\rangle\langle\sigma|| m\rangle\right|^{2} .
$$

Here $n_{F}(x)$ is the Fermi function, which depends on the temperature and chemical potential of the electron reservoir, $E_{n}$ and $E_{m}$ are the eigenstates of the QD Hamiltonian, and $\Gamma_{0}$ $=\Sigma_{k}\left|V_{k}\right|^{2} \rho\left(E_{f}\right)$ is the tunneling rate for the fermion in and out of the dot. The matrix elements featured in Eqs. (7) and (8) are strongly spin dependent and only connect states in which the Mn spin changes, at most, by one unit.

An important quantity in our simulations is the average charge $\langle q\rangle=\sum_{n=7,18} p_{n}(t)$. Since we consider an initially neutral dot, this quantity goes from zero to the steady-state occupation in a time scale given by $T_{1 Q} \equiv\left(\Gamma_{0}\right)^{-1}$. In this second set of simulations we measure time in units of $T_{1 Q}$. The evolution of the magnetization and the empty dot occupations on this case look very similar to those of Fig. 7. In particular, the magnetization decays exponentially in a time scale much longer than the charge relaxation time.

We have computed the Mn spin-relaxation time, $T_{1 M}$, as a function of the parameters of the simulation, $\Gamma_{0}, k_{B} T, J, \epsilon$, and $\langle q\rangle$. We have found that the crucial quantities are the average charge, the spin-flip anisotropy $\epsilon_{h}$, and $\Gamma_{0}$ as shown
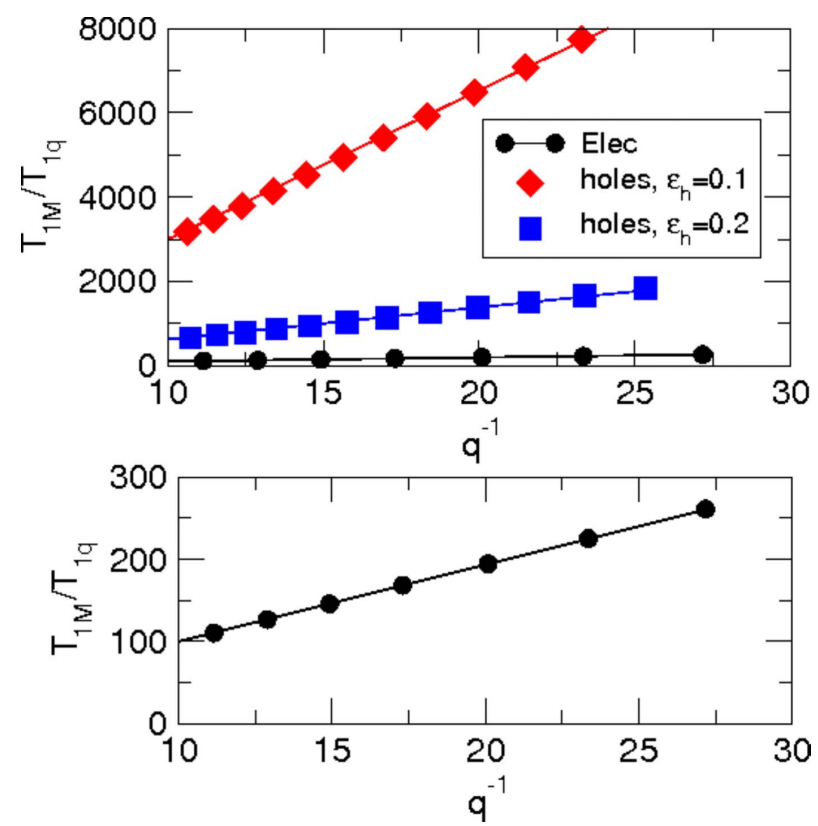

FIG. 8. (Color online) Mn spin-relaxation time, originated by charge fluctuations in the dot, as a function of average charge in the dot for electrons and holes with different $\epsilon_{h}$ values. The straight lines are linear fits of the numerical data.

in Fig. 8. To a very good approximation, we have observed that $\Gamma_{0} T_{1 M}$ depends linearly on $\langle q\rangle^{-1}$ for moderate values of $\langle q\rangle$. Thus, this mechanism shows a strong dependence on the density of carriers in the WL in agreement with our observations.

In Fig. 8 we see that in the case of tunneling electrons $(\epsilon=1)$, for an average charge of 0.1 , we have $T_{1 M}$ $\simeq 100 \Gamma_{0}^{-1}$. For tunneling holes with $\epsilon_{h}=0.2$ and $\langle q\rangle=0.1$ we have $T_{1 M} \simeq 600 \Gamma_{0}^{-1}$. Interestingly, these numbers are weakly dependent on the value of $J$ and on the temperature. In order to obtain an absolute number for $T_{1}^{M}$ we need an estimate of $\left(\Gamma_{0}\right)$ the charge scattering rate. An upper limit for $\Gamma_{0}$ is provided by the linewidth observed in the PL spectrum. Since the charge scattering would result in a broadening of the linewidth, we can infer that $\hbar \Gamma_{0}<50 \mu \mathrm{eV}$. Thus, using $\hbar$ $=0.65 \mathrm{meV}$ ps we have $T_{1}^{M}>600 \frac{\hbar}{\Gamma_{0}} \simeq 8 \mathrm{~ns}$ for $\langle q\rangle=0.1$. This is a lower bound for the spin-scattering time or an upper bound for the spin-scattering rate. Thus, single-hole tunneling events could relax the spin of the $\mathrm{Mn}$ in a time scale of 8 ns for a dot, which is charged $10 \%$ of the time. Agreement with the experimental result can be obtained by taking smaller average charge in the dot, smaller spin-flip interaction, or smaller tunneling rate $\Gamma_{0}$. We note that the proposed mechanism is similar to the hole spin-relaxation mechanism proposed by Smith et al. ${ }^{12}$ There, a spin-up hole tunnels out of the dot and a spin-down hole tunnels in resulting in an effective spin relaxation for the hole.

In summary, we have two mechanisms that account for carrier-induced $\mathrm{Mn}$ relaxation in the dot in a time scale of 10 ns. In both cases the dot must be considered as an open system. In the Korringa relaxation mechanism the Mn spin is exposed to extended WL states. In the charge-fluctuation mechanism the occupation of the dot state fluctuates due to 
its coupling to extended WL states. These two mechanisms are not exclusive and might operate at the same time. Whereas the Korringa mechanism is almost identical to that of $(\mathrm{Cd}, \mathrm{Mn}) \mathrm{Te}$ quantum wells, ${ }^{25}$ the charge-fluctuation mechanism is specific of quantum dots coupled to a continuum.

\section{CONCLUSION}

In conclusion, we used time-resolved and photoncorrelation measurements to probe the spin dynamics of a single magnetic atom (Mn) interacting with photocreated carriers at zero magnetic field. Fluctuations of the localized Mn spin control the statistics of the photons emitted by a single Mn-doped QD. The spin-relaxation time of the Mn atom around $20 \mathrm{~ns}$ appears to be particularly sensitive to the spin-spin coupling with free carriers injected in the QD or on the vicinity of the QD. The fast photoinduced spin relaxation of the Mn is dominated by spin and energy transfers from hot photocarriers directly to the Mn atom. Minimizing the injection of free carriers by optimizing the excitation conditions would make it possible to reach longer fluctuation times. As a consequence, a determination of the intrinsic Mn spin dynamics at zero magnetic field, ultimately limited by the spinlattice interaction, could only be performed in the absence of free or confined carriers.

\section{ACKNOWLEDGMENTS}

This work was supported by French ANR contracts MOMES and CoSin. J. Fernández-Rossier acknowledges funding from MEC-Spain (Grants FIS200402356 and HF2006-0004) and Consolider CSD2007-0010. *lucien.besombes@gernoble.cnrs.fr

${ }^{1}$ M. Xiao, I. Martin, E. Yablonovitch, and H. W. Jiang, Nature (London) 430, 435 (2004).

${ }^{2}$ J. Fernandez-Rossier and R. Aguado, Phys. Rev. Lett. 98, 106805 (2007).

${ }^{3}$ J. Lambe and C. Kikuchi, Phys. Rev. 119, 1256 (1960).

${ }^{4}$ M. K. Kneip, D. R. Yakovlev, M. Bayer, A. A. Maksimov, I. I. Tartakovskii, D. Keller, W. Ossau, L. W. Molenkamp, and A. Waag, Phys. Rev. B 73, 045305 (2006).

${ }^{5}$ T. Dietl, P. Peyla, W. Grieshaber, and Y. Merle d'Aubigne, Phys. Rev. Lett. 74, 474 (1995).

${ }^{6}$ A. J. Heinrich, J. A. Gupta, C. P. Lutz, and D. M. Eigler, Science 306, 466 (2004).

${ }^{7}$ D. Kitchen, A. Richardella, J.-M. Tang, M. E. Flatte, and A. Yazdani, Nature (London) 442, 436 (2006).

${ }^{8}$ L. Besombes, Y. Léger, L. Maingault, D. Ferrand, H. Mariette, and J. Cibert, Phys. Rev. Lett. 93, 207403 (2004).

${ }^{9}$ L. Maingault, L. Besombes, Y. Léger, C. Bougerol, and H. Mariette, Appl. Phys. Lett. 89, 193109 (2006).

${ }^{10}$ C. Couteau, S. Moehl, F. Tinjod, J. M. Gérard, K. Kheng, H. Mariette, J. A. Gaj, R. Romestain, and J. P. Poizat, Appl. Phys. Lett. 85, 6251 (2004).

${ }^{11}$ Y. Léger, L. Besombes, L. Maingault, D. Ferrand, and H. Mariette, Phys. Rev. B 72, 241309(R) (2005).

${ }^{12}$ J. M. Smith, P. A. Dalgarno, R. J. Warburton, A. O. Govorov, K. Karrai, B. D. Gerardot, and P. M. Petroff, Phys. Rev. Lett. 94, 197402 (2005).

${ }^{13}$ A. Kiraz, S. Falth, C. Becher, B. Gayral, W. V. Schoenfeld, P. M. Petroff, L. Zhang, E. Hu, and A. Imamoglu, Phys. Rev. B 65, 161303(R) (2002).

${ }^{14}$ A. O. Govorov and A. V. Kalameitsev, Phys. Rev. B 71, 035338 (2005).

${ }^{15}$ Y. Léger, L. Besombes, L. Maingault, and H. Mariette, Phys. Rev. B 76, 045331 (2007).

${ }^{16}$ D. H. Feng, I. A. Akimov, and F. Henneberger, Phys. Rev. Lett. 99, 036604 (2007)
${ }^{17}$ L. M. Woods, T. L. Reinecke, and R. Kotlyar, Phys. Rev. B 69, 125330 (2004).

${ }^{18}$ H. Dery, L. Cywinski, and L. J. Sham, Phys. Rev. B 73, 161307(R) (2006).

${ }^{19}$ A. Vasanelli, R. Ferreira, and G. Bastard, Phys. Rev. Lett. 89, 216804 (2002).

${ }^{20}$ M. G. Tyazhlov, A. I. Fillin, A. V. Larionov, V. D. Kulakovskii, D. R. Yakovlev, A. Waag, and G. Landwehr, JETP 85, 784 (1997).

${ }^{21}$ D. Scalbert, J. Cernogora, and C. Benoit la Guillaume, Solid State Commun. 66, 571 (1988).

${ }^{22}$ L. Besombes, Y. Leger, L. Maingault, D. Ferrand, H. Mariette, and J. Cibert, Phys. Rev. B 71, 161307(R) (2005).

${ }^{23}$ J. K. Furdyna, J. Appl. Phys. 64, R29 (1988).

${ }^{24}$ J. Lambe and C. Kikuchi, Phys. Rev. 119, 1256 (1960); D. Scalbert, J. Cernogora, and C. Benoit a la Guillaume, Solid State Commun. 66, 571 (1988).

${ }^{25}$ B. Konig, I. A. Merkulov, D. R. Yakovlev, W. Ossau, S. M. Ryabchenko, M. Kutrowski, T. Wojtowicz, G. Karczewski, and J. Kossut, Phys. Rev. B 61, 16870 (2000).

${ }^{26}$ E. Souto, O. A. C. Nunes, and A. L. A. Fonseca, Solid State Commun. 129, 605 (2004).

${ }^{27}$ Y. Léger, L. Besombes, J. Fernández-Rossier, L. Maingault, and H. Mariette, Phys. Rev. Lett. 97, 107401 (2006).

${ }^{28}$ J. Fernández-Rossier, Phys. Rev. B 73, 045301 (2006).

${ }^{29}$ J. Fernández-Rossier and L. Brey, Phys. Rev. Lett. 93, 117201 (2004).

${ }^{30}$ Claude Cohen-Tannoudji, Jacques Dupont-Roc, and Gilbert Grynberg, Atom-Photon Interactions: Basic Processes and Applications (Wiley, New York, 1992).

${ }^{31}$ M. Qazzaz, G. Yang, S. H. Xin, L. Montes, H. Luo, and J. K. Furdyna, Solid State Commun. 96, 405 (1995).

${ }^{32}$ P. A. Dalgarno, M. Ediger, B. D. Gerardot, J. M. Smith, S. Seidl, M. Kroner, K. Karrai, P. M. Petroff, A. O. Govorov, and R. J. Warburton, Phys. Rev. Lett. 100, 176801 (2008). 